\title{
Determination of Genetic Characterization and Circulation Pattern of Respiratory Syncytial Virus (Rsv) in Children With a Respiratory Infection, Tehran, Iran, During 2018-2019
}

\section{Forough Tavakoli}

Tehran University of Medical Sciences

Anahita Izadi

Tehran University of Medical Sciences

Jila Yavarian

Tehran University of Medical Sciences

Ali Sharifi-Zarchi

Sharif University of Technology

Vahid Salimi

Tehran University of Medical Sciences

Talat Mokhtari-azad ( $\nabla$ f-tavakoli@razi.tums.ac.ir )

Tehran University of Medical Sciences https://orcid.org/0000-0002-6556-4072

\section{Research Article}

Keywords: Respiratory Syncytial Virus, genotype, Phylogenetic analysis, genetic diversity, Hospitalization

Posted Date: July 29th, 2021

DOI: https://doi.org/10.21203/rs.3.rs-742648/v1

License: (c) (i) This work is licensed under a Creative Commons Attribution 4.0 International License.

Read Full License 


\section{Abstract}

The RSV-associated disease accounts for a significant health burden particularly in infants and young children who need to be hospitalized. Since continuous surveillance of circulating RSV genotypes is critical worldwide, this study aimed to investigate the genetic diversity of RSV circulating strains causing upper or lower acute respiratory infection. Our attention was geared towards studying the cases in hospitalized or outpatient children younger than 2 years of age in Iran during 2018/2019. In this study, nasopharyngeal aspirates collected from 206 children who presented with respiratory infection symptoms, were admitted to the referral pediatric ward of Bahrami children's hospital in Tehran, Iran. RSV-positive samples were detected via Nested RT-PCR. The glycoprotein gene was sequenced, and virus genotypes were confirmed through phylogenetic analysis by the MEGA X program. A total of 74(35.92\%) samples tested positive for RSV. Among them, sequencing was done in 10 specimens from 2018 (RSV-A: RSV-B =4:6) and 19 specimens from 2019 (RSV-A: RSV-B =16:3). According to Phylogenetic analysis, all RSV-A strains were assigned as ON1 genotype and RSV-B strains were assigned as BA9 genotype. A new $\mathrm{N}$-glycosylation site in Iranian BA9 and positive selection in ON1 genotype was observed. Phylogenetic characterization of strains in the current study revealed co-circulation of ON1 and BA9 as the only prevalent genotypes of both RSV-A and -B groups.

\section{Introduction}

The overwhelming majority of acute lower respiratory infections (ALRI) and hospitalization in early life are caused by the Respiratory syncytial virus_(RSV) (Shi et al. 2020). Newborn infants and young children and people aged $>65$ years are more susceptible to develop severe RSV infection (Smithgall et al. 2020). RSV infection usually shows mild, cold-like symptoms that recover in a short period, but young children may develop pneumonia or bronchiolitis and it may sometimes cause death (Garcia-Mauriño et al. 2019; Fergie et al. 2020). Mortality rates in infants hospitalized with RSV are approximately 10 times greater than in infants infected with the influenza virus (Miyairi and DeVincenzo 2008).

The biological mechanisms that cause disease progression remain to be fully understood (Janssen et al. 2007). However, multiple contributing factors including environment, pathogen, and the host's heredity determine susceptibility to pathogens and also the course of the disease (Miyairi and DeVincenzo 2008).

RSV is a species of Orthopneumovirus genus within the Pneumoviridae family (Cui et al. 2013). The genome of RSV is a negative-sense ssRNA of about $15.2 \mathrm{~kb}$, that contains 10 genes encoding 11 proteins (Malasao et al. 2015). Three surface proteins of virion are coded by attachment (G), fusion (F), and small hydrophobic (SH) genes (Gimferrer et al. 2015). The G glycoprotein is a highly glycosylated type II surface protein (Tabor et al. 2020). It contains two hypervariable (HVR) regions on the extracellular domain (HVR1 and HVR2), which are separated by a 13-amino acid highly conserved region (aa 163189) (Zhang et al. 2018). The region used for Genotyping and evolution analysis is the HVR2 in the Cterminal (Tan et al. 2012). 
The $G$ protein of Human RSV is separated based on antigenic differences within it into two antigenic subgroups, RSV-A and RSV-B (Marlene Ihling et al. 2021). Both RSV-A and -B subgroups are classified further to the 14 (GA1-7, SAA1, NA1-4, ON1, and CB-A) and 25 genotypes (GB1-4, BA1-12, SAB1-4 URU1-2, BA-C, CB-1, and CB-B) respectively, according to sequence variation of the HVR-2 of the $G$ gene (Korsun et al. 2021).

ON1, the novel genotype that first emerged in 2010 in Ontario Canada, rapidly became the dominant genotype of the RSV A group worldwide (Malasao et al. 2015; Kang et al. 2020). ON1 has 72 nucleotide duplications in the HVR-2 of the G gene (Trento et al. 2003) and resembles the BA genotype that contains 60-nucleotide duplications in the HVR-2 of the G gene (Dapat et al. 2010).

Our knowledge about the molecular phylogeny of RSV in Iran is insufficient. GA-1, GA-2, GA-5, and BA were the genotypes co-circulating in Iran from 2007 to 2013 (Salimi et al. 2016). In 2015-2016 a shift to ON-1 as the main circulating genotype occurred (Malekshahi et al. 2019). So, continuing molecular and epidemiological surveillance of the HRSV strains is needed to provide a better understanding of the virus pathogenesis and vaccine designs. Here, findings of genetic characterization of the HRSV strains circulating in Iran during 2018/2019 are reported.

\section{Material And Methods}

\subsection{Patients and samples}

In total, 206 nasopharyngeal swabs were taken from children under two years of age from January to March 2018 and December 2018 to May 2019 in the referral pediatric ward of Bahrami children's hospital in Tehran, Iran. The patients had symptoms ranging from mild to severe. Our cases were grouped as inpatient and outpatient. Demographic information of the studied cases is shown in table1.

Informed consent was obtained from all parents of infants following a description of the aim of the study. All samples were taken by Copan swabs containing transport media and were rapidly transported to the Virology Department, Tehran University of Medical Sciences.

\subsection{Viral RNA extraction, Reverse transcription \& HRSV detection}

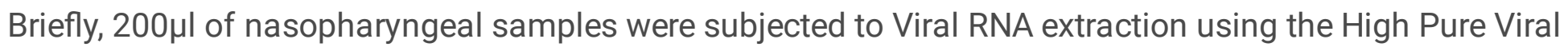
Nucleic Acid Kit (Roche, Mannheim, Germany), according to the manufacturer's instruction and stored at $-80^{\circ} \mathrm{C}$ for the next steps. Reverse transcription was performed on extracted RNA using First-strand cDNA synthesis kit (Roche, Mannheim, Germany) according to the manufacturer's instruction. For the HRSV detection, a Hemi-nested polymerase chain reaction of the $\mathrm{N}$ gene was done as previously described (Malekshahi et al. 2010). The following primer sets used (Kawabuchi-Kurata et al. 2014) are indicated in table 2.

\subsection{Genotyping \& DNA sequencing}


HVR2 region of the $G$ gene was amplified using a Hemi-nested PCR as previously reported (Malekshahi et al. 2020) employing the primer pairs in Sato et al.'s method(Sato et al. 2005) which detected both the RSV A and B types (table2). To determine if the genotypes of RSV strains tested positive, the PCR products were sequenced using the ABI BigDye ${ }^{\circledR}$ Terminator Cycle Sequencing Kit v3.1, on the 3130 Genetic Analyzer Automated Sequencer (Applied Biosystems Foster City, California, USA).

\subsection{Phylogenetic analysis of the $G$ gene sequences}

To determine the genotype of the detected viruses, sequences were separately aligned to reference sequences of the genotypes in RSV-A, and -B groups. Next, a phylogenetic tree of RSV-A strains was constructed using the maximum-likelihood method and the Tamura-Nei (TN93) model. The best substitution model was obtained by finding the best DNA/Protein models option implemented in MEGA X software. Subsequently, the reliability of the phylogenetic tree was evaluated by the 1000 bootstrap replicate with a cut-off of $70 \%$. The Phylogenetic tree of RSV-B strains was constructed using the neighbor-joining method and the Tamura-Nei model. To determine the evolutionary distances between Iranian strains and strains circulating in other regions, another phylogenetic tree was drawn by including strains of related genotypes from other countries in the GenBank. Using the maximum-likelihood method and Tamura Nei-model were utilized for the ON1 strains, while the maximum-likelihood method and HKY model were employed for BA9 strains. The nomenclature of detected viruses was based on the method described by Salimi V et al. (Salimi et al. 2021) as follows: HRSV/subgroup/geographic identifier/unique identifier/year of collection (e.g., HRSVs/A/Tehran. IRN/ 8/2018). The sequences are available from GenBank under accession numbers: MW675694- MW675722.

\subsection{Amino acid analysis:}

To assay the possible synonymous mutations, amino acid sequences of the $\mathrm{G}$ gene of Iranian strains were subjected to multiple sequence alignments with corresponding reference sequences utilizing the Bio Edit v.7.2.5 software (Fig3). Moreover, the proportion of differences (p-distance) of both nucleotide and amino acid sequences were evaluated.

\section{$2.6 \mathrm{~N}-/ 0$-glycosylation sites analysis:}

The acquisition or loss of putative $\mathrm{N}$ - and O-glycosylation sites in the HVR2 region of the G protein were predicted by using NetN-Glyc 1.0 (www.cbs.dtu.dk/services/NetNGlyc) and NetOGlyc 4.0 Servers (www.cbs.dtu.dk/services/NetOGlyc) respectively, with a threshold value of 0.5 .

\subsection{Selection pressure analyses:}

The synonymous (dS) and nonsynonymous ( $d N$ ) mutation rates determine whether any sites in the $G$ gene are subjected to Selection pressure. For interpretation, the $d N>d S$ represent the index of positive selection, while $\mathrm{dN}<\mathrm{dS}$ represent the negative pressure. Analyses were carried out on the Datamonkey website interface (http://www.datamonkey.org) (Pond and Frost, 2005) using the following methods: SLAC (single likelihood ancestor counting), FEL (fixed effects likelihood), MEME (mixed effects model for 
evolution) with a p-value threshold of 0.1 and FUBAR (Fast, Unconstrained Bayesian AppRoximationa) with a p-value threshold of 0.9 .

\section{Results}

\subsection{Patient characteristics}

From January to March 2018, and December 2018 to May 2019, 206 nasopharyngeal swabs were collected from children (33.98\% female, $66.01 \%$ male, 0 month-2 years) with upper or lower RTI. The age of the cases ranged from 1 month to 24 months. The mean ( \pm S.D.) age of the studied cases was $6.5 \pm 5.9$ (months) and a median age of 5.00 months. Among the study patients, $110(53.39 \%)$ were less than 6 months old; 62 (30.09\%) were between 6 and 11 months old, and 34(16.50\%) were between 12 and 24 months old. The study comprised 148 inpatients (71. 84\%) and 58 outpatients (28.15\%).

\subsection{Prevalence of RSV from 2018-2019}

Out of the 206 nasopharyngeal swabs, a total of 74(35.92\%) patients were positive for RSV. Common symptoms in RSV-positive patients were fever (80\%), cough (49.3\%), bronchiolitis (67.1\%), sneezing (49.3), nasal congestion (82.2), and rhinorrhea (60.2). Among the RSV-positive cases, $75.67 \%$ were male and $24.32 \%$ were female. The RSV positivity rate in males was significantly higher than in females (OR $=$ $1.65,95 \% \mathrm{Cl}: 1.05-2.59 ; \mathrm{P}=0.01)$. Children under 6 months were most significantly affected by RSV (41.1\%) ( $P=0.01)$. Among all RSV positive samples, 29 samples were successfully sequenced of which, 19 (65.51\%) samples were identified as RSV-A, and 9 (31.03\%) as RSV-B. 4 RSV-A and 6 RSV-B sequences from the samples were collected in the 2018 seasonal epidemic, and 16 RSV-A and 3 RSV-B sequences from the samples were collected in the 2019 seasonal epidemic.

Between 2015 and 2016, RSV-A was reported as the only RSV type circulating in Tehran. However, this study shows the co-circulation of both RSV-A and -B types from 2018 to 2019. Among the 10 RSV positive samples subjected to sequencing in $2018,6(60 \%)$ samples were RSV-B. In 2019, the type dominance shifted to RSV-A (RSV-A: RSV-B $=84.21 \% ; 15.78 \%$ ). The peak of the RSV detection in both consecutive 2018-2019 seasons was recorded in March $(P<0.01)$ followed by February. The monthly distribution of RSV strains is indicated in Fig 1.

\subsection{Phylogenetic analysis of RSV genotypes}

According to Phylogenetic analysis, all RSV-A strains were classified as the ON1 genotype (Fig2). The phylogenetic tree of $\mathrm{ON}-1$ strains in various parts of the world is clustered in two different lineages (Figure 3). Even though the Iranian strains are clustered in both lineages, most of them, however, are grouped in Lineage I. Strains in lineage I, are clustered with closely related strains from Lebanon, Egypt, Saudi Arabia, Thailand, India, Italy, Germany, USA, Australia, and Greece. Lineage II consists only of our isolates which are clustered separately. 
Based on Phylogenetic analysis, all RSV-B strains were classified as the BA9 genotype (Fig2). The phylogenetic analysis of Iranian sequences and BA9 strains of various parts of the world is shown in Figure 3. The phylogenetic tree of BA9 strains in various parts of the world is clustered in tow lineages. All BA9 Iranian strains fall into lineage I, clustered with closely related strains from Bulgaria, Korea, India, China, Taiwan, and Russia. At the nucleotide level, the average pairwise distances within the ON1 genotype in our strains was 0.04 . In addition, the sequenced p-distance of our ON1 strains compared to the ON1 prototype strain (JN257693) was in the range of 0.01 to 0.05 . The average pairwise distances within the BA9 genotype in our strains was 0.01 . The nucleotide distances between the study strains and prototype BA-9 strain (DQ227395.1) were in the range of 0.04 to 0.06 .

\subsection{Amino acid analysis}

Compared to the ON1 prototype (JN257693.1), 17 amino acid substitutions were detected in more than 2 Tehran strains, of which L274P, L298P, Y304H, and L310P were the most common substitutions. The position of the first three of these substitutions is in the insertion site or its duplicated site. Six unique substitutions were detected including E224G ( $n=2)$, L226P ( $n=2)$, T231S ( $n=2), N 242 S(n=2)$, S277P $(n=4)$ and $\mathrm{Y309H}(\mathrm{n}=4)$. An association was observed between substitutions of E224G and T231S, as well as S2770P and Y309H. 2 amino acid sites, L226P and G284S, subjected to substitutions in the Iranian strains (RSVs/A/Tehran.IRN/53/2019, RSVs/A/Tehran.IRN/61/2019, and RSVs/A/Tehran.IRN/11/2018) were detected as an epitope in escape-mutant strains. These two sites plus 225A are predicted to be putatively positively selected. Compared to the BA reference strain (AY333364.1), five substitutions were found in BA9 studied strains as characteristics of the BA9 genotype: L223P, S247P, T270I, V271A, and $\mathrm{H} 287 \mathrm{Y}$. Among them, V271A is specific to the BA9 genotype consistent with a previous report (Dapat et al. 2010). Compared to the BA-9 reference strain (DQ227395.1), four substitutions occurred in all nine studied strains including T254I, I281T, T290I, and T312I which seemed to be characteristic of a separate sub-lineage. Seven unique substitutions were detected in individual strains including T228A $(n=1), 1229 \mathrm{~L}$ $(n=1)$, T250A $(n=1)$, P295L $(n=1)$, S307P $(n=1)$, T308S $(n=1)$ and S311C $(n=2)$. Interestingly, the mutation in the stop codon (Stop 313 Q) was found in Iranian strains circulating in different cities from 2009 to 2016 as well as in Iraq, Turkey, the Philippines, and Belgium. This substitution transformed the stop codon into a coding site in position 313. As codon 313 in the BA prototype (AY333364.1) encodes an amino acid, this constitutes a reverse substitution called a flip-flop pattern.

\section{$3.5 \mathrm{~N}-/ 0$-glycosylation sites analysis:}

The pattern of N-glycosylation of the HVR2 region in all RSV-A studied strains was the same as the ON1 prototype and included a potential $\mathrm{N}$-glycosylation site at positions 237 . The potential $\mathrm{N}$-glycosylation site in the BA9 reference sequence is in position 296. In all our strains this $\mathrm{N}$-glycosylation motif was conserved. Besides, P231L substitution in four of the studied strains resulted in a potentially new Nglycosylation site. Some Ser/Thr amino acids in the HVR2 region of both ON1 and BA9 strains were substituted with other amino acids that resulted in the loss of the 0-glycosylation sites. That notwithstanding, some other substitutions resulted in the acquisition of 0-glycosylation sites. 


\subsection{Selection pressure analyses}

Using MEME, FUBAR, and FEL Methods, evidence of positive or diversifying selection of ON1 strains at site 262 was observed. Moreover, strains were subjected to pervasive negative or purifying selection in 230 and 239 codons using FUBAR, FEL, and SLAC. Selection analyses of BA9 strains exhibited sites 311 as positive or diversifying ( $p=0.92)$, and 296 and 304 as negative or purifying selections $(p=0.92)$ using the FUBAR method. Other methods did not find this positive and negative selection. Since a consensus of at least two models is essential to confirm the selection pressure sites (Haider et al. 2018) we do not consider them as selection evidence.

\section{Discussion}

In this study, we determined the molecular epidemiology and genetic diversity of RSV circulating strains among children aged less than 2 years with acute RTI in Iran over two consecutive seasons between 2018- 2019. The prevalence rate of RSV infection (36\%), was lower than those for the 2015-2016 study by 46.2\%, but higher than those for other developing countries (Auksornkitti et al. 2014; Malasao et al. 2015; Abou-El-Hassan et al. 2019; Aamir et al. 2020; Marlene lhling et al. 2021). The variation in the incidence rate of RSV infection from year to year and country to country can be explained in part by factors such as population study, sampling, diagnostic method, climate, and other factors(Zhang et al. 2015; Korsun et al. 2021). RSV prevalence peaked in March with more than half of positive the cases, followed by February in both 2018 and 2019 seasons, which support the previous findings in Iran during 2015-2016 with a peak in January. It describes the constancy in RSV seasonality in Iran. The exact beginning, peak, and end of the RSV epidemic pattern may be variable from year to year (19 January 2018) depending on climate (Tang and Loh 2014) and geographical regions. The current study revealed that both the subgroups RSV-A and -B circulate in the Iranian population. All the 20 sequenced RSV-A from the 20182019 seasons were assigned as the ON1 genotype and all the 9 sequenced RSV-B were categorized into the BA9 genotype. Regarding the sequenced samples, RSV-B dominated over RSV-A during 2018 (60\%). During the 2019 season, RSV-B prevalent decreased down to $15.78 \%$ and RSV-A predominated by $84.21 \%$. According to previous reports in Iran, fluctuation in the circulation of both subgroups and genotypes of RSV were observed and are consistent with those of other studies (Malekshahi et al. 2010; Faghihloo et al. 2011b; Korsun et al. 2017). During the 2007-2008 period, the prevalence of GA1, GA2, and GA5 was reported by Faghihloo et al. (Faghihloo et al. 2011a). The same group reported GA1, GA2, and BA genotypes during 2009-2013 (Faghihloo et al. 2011b), while Malekshahi et al. reported ON1 genotype as the only RSV genotype in 55 cases from 2015 to 2016 (Malekshahi et al. 2019). This suggests that the RSV-A, GA1, and GA2 genotypes prevalent in Iran have been replaced by ON1 since 2016. Further, RSV-B which had not been detected during 2015-2016 in Tehran, re-emerged during 20182019 as a BA9 genotype. Alternating prevalence is likely due to herd immunity against circulating RSV subgroups or genotypes. However, the $\mathrm{G}$ gene variations may promote the escape of the pre-existing host immune response (Melero and Moore 2013), which poses a challenge for vaccine development. Since the first ON1 emergence in Iran in 2015, it has been dominated and is recently known as the only RSV-A genotype of RSV-A. This is in line with recent reports around the world (Zheng et al. 2017; Abou-El-Hassan 
et al. 2019; Kang et al. 2020). The BA9 genotype was first detected in Niigata, Japan during the 20062007 season and is known as the most prevalent RSV-B genotype in nearly all countries. Hotard et al. indicated that duplication in the BA strain-enhanced virus attachment and fitness(Hotard et al. 2015), resulted in selective advantage and predominance. Binding enhancement may also contribute towards the survival of ON1 strains although this needs further analysis. Based on the phylogenetic tree of RSV-A ON1 (Figure 2), there were 2 lineages in Iran. Most of the studied sequences were in lineage I along with sequences from across the world. For example, Lebanon, Egypt, Saudi Arabia, Thailand, India, Italy, Germany, and Greece. Lineage II included 2 Iranian strains which were not clustered with any of the ON1 strains reported from other countries. Previously ON1 circulating strains in Iran in 2015-2016 were closely related to our studied strains. One of our strains clustered to the Lebanese strains of 2016-17 which shared seven substitutions compared to other studied strains. These were G284S, E295K, P320K, Y304H, S314P, T319I, and P320K with bootstrap support of 98 and a p-distance $<0.001$, considered by Abou-El et al. as a new genotype [32]. Contrarily, this did not appear as a separate genotype in our phylogenetic tree. Some amino acid changes of the Iranian strains which were found nearly in all samples detected in the initial emergence of the ON1 genotype, comprised of the L274P, L298P, L310P, and Y304H substitutions. In addition to the mentioned changes, unique substitutions were detected in our isolates; E224G, L226P, T231S, N242S, S277P, and Y309H that may be specific to the Iranian strains. This may be monitored in the future as it occurred in a previous unique change in Iran; the K321E, which was also identified in the current study. Another previous unique change was the $1243 \mathrm{~S}$ which was not only detected in the current study but also seen in Italian isolates in 2020. Given that multiple epitopes-neutralizing antibodies interactions in the hypervariable $C$ terminal region of the $G$ gene occurs (Cane 1997), substitutions in this region indicate the antigenicity variation due to selection pressure (Kenmoe et al. 2018). According to Figure 3, all BA9 Iranian isolates were assigned to lineage I and were closely related to strains detected since 2017 in various parts of the world. This included Bulgaria, Korea, India, China, Taiwan, and Russia showing a broad geographic spread. These strains are in a separate sub-lineage with specific substitutions; T254I, I281T, T290I, T312I. Besides, BA9 strains circulating in different cities in Iran from 2009 to 2016 clustered in separate lineages compared to our strains. The mutation in the stop codon (Stop 313 Q) that was detected in the strains circulating in different cities of Iran from 2009 to 2016 as well as in Iraq, Turkey, the Philippines, and Belgium, altered the length of the $G$ protein from 313 amino acids to 320 amino acids. No study has yet been found that examines the effect of this change on the pathogenicity of the virus. The N-Glycosylation pattern of ON1 Iranian strains was the same as the prototype ON1 strain in codon 237. Also, in BA9 strains the N-Glycosylation site was the same as the BA9 prototype in codon 296. However, in four of the BA9 strains, a P231L substitution resulted in the new Nglycosylation site. This substitution is also present in other countries such as India, Thailand, China, Korea, Taiwan, Myanmar, Cameroon, and Bulgaria. Given that Glycosylation accounts as one of the important viral strategies for evasion of pre-existing antibodies (Cui et al. 2013), this substitution probably changes the antigenicity of strains and will be selected through the evolution. 231L NGlycosylation site has also been detected in the BA-10 genotype strains (Kenmoe et al. 2018). The G protein of the $\mathrm{ON} 1$ isolates is under positive selective pressure at site 262, as described earlier (Yoshihara et al. 2016; Zheng et al. 2017). This site is subjected to E262K substitution in some of our isolates as 
detected in other regions such as Spain, Vietnam, China, Italy, and Portugal. The evolution analysis also revealed 2 negatively selected sites. The G protein of the BA-9 isolates is under positive selective pressure at site 312 using the FUBAR method, and under negative or purifying selection pressure at the 225,297 , and 305 sites. These selected sites in Iranian isolates are not supported by other methods. Therefore, these results are not strongly confirmed and were not found by other studies. A possible explanation for this may be the lack of any evolutionary study in the strains carrying this T312l, which was observed in strains detected around 2017 onwards This may be categorized as a separate sub-lineage containing selected sites showing the selection pressure from the host. The current study only examined a phylogenetic analysis of isolates successfully sequenced. Some samples which tested positive for the $\mathrm{N}$ gene were negative for the $\mathrm{G}$ gene. It may be due to low RNA concentration. As genotyping is based on the $\mathrm{G}$ gene, samples that tested negative or weakly positive for the $\mathrm{G}$ gene were not sequenced. The low RNA concentration may have resulted from either RNA degradation or improper sampling.

\section{Conclusion}

In summary, this study demonstrated that the RSV prevalence among children under the age of 2 was $36 \%$ with co-circulation of ON1 and BA9 genotypes in Tehran, the capital of Iran. Continuing Phylogenetic and molecular surveys is critical for tracking epidemic-circulating strains, and the correlation between distinct genotypes and pathogenesis to develop effective prophylactic and therapeutic interventions. Further studies in all regions of Iran with more focus on the F gene or whole genome sequences along with the $\mathrm{G}$ gene via Next Generation Sequencing are strongly recommended to give a more comprehensive insight on the circulating RSV strains.

\section{Declarations}

\section{Acknowledgment:}

The authors appreciate the School of Public Health, Tehran university of Medical Science for providing financial support. The authors also gratefully acknowledge Professor. Louis Bont for providing the Copan swabs for the nasopharyngeal sample collection.

\section{Funding Information}

This study was extracted from Ph.D. Dissertation. This work received grant from School of Public Health, Tehran university of Medical Science (grant number: 97-03-27-39065)

\section{Competing Interest:}

The author(s) declare that there are no conflicts of interest.

\section{Availability of data and material:}

Data is available on the request. 


\section{References}

Respiratory Syncytial Virus Seasonality - United States, 2014-2017

https://www.cdc.gov/mmwr/volumes/67/wr/mm6702a4.htm (14 June).

Aamir UB et al. (2020) Molecular characterization of circulating respiratory syncytial virus genotypes in Pakistani children, 2010-2013. Journal of infection and public health 13:438-445

Abou-El-Hassan H et al. (2019) Detection of ON1 and novel genotypes of human respiratory syncytial virus and emergence of palivizumab resistance in Lebanon. Plos one 14:e0212687

Auksornkitti V et al. (2014) Molecular characterization of human respiratory syncytial virus, 2010-2011: identification of genotype ON1 and a new subgroup B genotype in Thailand. Archives of virology 159:499-507

Cane PA (1997) Analysis of linear epitopes recognised by the primary human antibody response to a variable region of the attachment $(G)$ protein of respiratory syncytial virus. Journal of medical virology 51:297-304

Cui $G$ et al. (2013) Genetic variation in attachment glycoprotein genes of human respiratory syncytial virus subgroups a and B in children in recent five consecutive years. PLoS One 8:e75020

Dapat IC et al. (2010) New genotypes within respiratory syncytial virus group B genotype BA in Niigata, Japan. Journal of clinical microbiology 48:3423

Faghihloo E, Rezaie F, Salimi V, Naseri M, Mamishi S, Mahmoodi M (2011a) Molecular epidemiology of human respiratory syncytial virus in Iran. Acta Virol 55:81-83

Faghihloo E et al. (2011b) Genetic diversity in the $G$ protein gene of human respiratory syncytial virus among Iranian children with acute respiratory symptoms. Iranian journal of pediatrics 21:58

Fergie J, Suh M, Jiang X, Fryzek JP, Gonzales T (2020) Respiratory Syncytial Virus and All-Cause Bronchiolitis Hospitalizations Among Preterm Infants Using the Pediatric Health Information System (PHIS). The Journal of Infectious Diseases

Garcia-Mauriño C et al. (2019) Viral load dynamics and clinical disease severity in infants with respiratory syncytial virus infection. The Journal of infectious diseases 219:1207-1215

Gimferrer L et al. (2015) Molecular epidemiology and molecular characterization of respiratory syncytial viruses at a tertiary care university hospital in Catalonia (Spain) during the 2013-2014 season. Journal of Clinical Virology 66:27-32

Haider MSH et al. (2018) BA9 lineage of respiratory syncytial virus from across the globe and its evolutionary dynamics. PloS one 13:e0193525 
Hotard AL, Laikhter E, Brooks K, Hartert TV, Moore ML (2015) Functional analysis of the 60-nucleotide duplication in the respiratory syncytial virus Buenos Aires strain attachment glycoprotein. Journal of virology 89:8258-8266

Janssen R et al. (2007) Host transcription profiles upon primary respiratory syncytial virus infection. Journal of virology 81:5958

Kang HM, Park KC, Park J, Kil HR, Yang EA (2020) Circulating Respiratory Syncytial Virus Genotypes and Genetic Variability of the G Gene during 2017 and 2018/2019 Seasonal Epidemics Isolated from Children with Lower Respiratory Tract Infections in Daejeon, Korea. Journal of Korean Medical Science 35

Kawabuchi-Kurata T, Misaki T, Suehiro Y, Komano AJ, Kase T, Takahashi K (2014) Longitudinal study on respiratory viral co-infections in the presence or absence of clinical manifestation in infants aged 0-2 years. Japanese journal of infectious diseases 67:216-220

Kenmoe S et al. (2018) Genetic diversity of human respiratory syncytial virus isolated among children with acute respiratory infections in southern Cameroon during three consecutive epidemic seasons, 2011-2013. Tropical medicine and health 46:1-8

Korsun N et al. (2021) Predominance of ON1 and BA9 genotypes of respiratory syncytial virus (RSV) in Bulgaria, 2016-2018. Journal of Medical Virology 93:3401-3411

Korsun $\mathrm{N}$ et al. (2017) Prevalence and genetic characterisation of respiratory syncytial viruses circulating in Bulgaria during the 2014/15 and 2015/16 winter seasons. Pathogens and global health 111:351-361

Malasao R et al. (2015) Molecular characterization of human respiratory syncytial virus in the Philippines, 2012-2013. PloS one 10:e0142192

Malekshahi SS, Azad TM, Yavarian J, Shahmahmoodi S, Naseri M, Rezaei F (2010) Molecular detection of respiratory viruses in clinical specimens from children with acute respiratory disease in Iran. The Pediatric infectious disease journal 29:931-933

Malekshahi SS et al. (2019) Molecular characterization of the glycoprotein and fusion protein in human respiratory syncytial virus subgroup A: Emergence of ON-1 genotype in Iran. Infection, Genetics and Evolution 71:166-178

Malekshahi SS et al. (2020) Genetic characterization of G protein in respiratory syncytial virus ON-1 genotype in Tehran. Future Virology 15:725-734

Marlene Ihling C et al. (2021) Molecular epidemiology of Respiratory Syncytial Virus in children in subSaharan Africa. Tropical Medicine \& International Health

Melero JA, Moore ML (2013) Influence of respiratory syncytial virus strain differences on pathogenesis and immunity. Challenges and Opportunities for Respiratory Syncytial Virus Vaccines:59-82 
Miyairi I, DeVincenzo JP (2008) Human genetic factors and respiratory syncytial virus disease severity. Clinical microbiology reviews 21:686

Salimi V, Tavakoli-Yaraki M, Yavarian J, Bont L, Mokhtari-Azad T (2016) Prevalence of human respiratory syncytial virus circulating in Iran. Journal of infection and public health 9:125-135

Salimi V et al. (2021) Proposal for Human Respiratory Syncytial Virus Nomenclature below the Species Level. Emerging Infectious Diseases 27:1-9

Sato $\mathrm{M}$ et al. (2005) Molecular epidemiology of respiratory syncytial virus infections among children with acute respiratory symptoms in a community over three seasons. Journal of Clinical Microbiology 43:36

Shi T et al. (2020) Global disease burden estimates of respiratory syncytial virus-associated acute respiratory infection in older adults in 2015: a systematic review and meta-analysis. The Journal of infectious diseases 222:S577-S583

Smithgall M et al. (2020) Epidemiology, clinical features, and resource utilization associated with respiratory syncytial virus in the community and hospital. Influenza and other respiratory viruses 14:247256

Tabor DE et al. (2020) Global molecular epidemiology of respiratory syncytial virus from the 2017- 2018 INFORM-RSV study. Journal of Clinical Microbiology 59

Tan L et al. (2012) Genetic variability among complete human respiratory syncytial virus subgroup A genomes: bridging molecular evolutionary dynamics and epidemiology. PloS one 7:e51439

Tang JW, Loh TP (2014) Correlations between climate factors and incidence-a contributor to RSV seasonality. Reviews in medical virology 24:15-34

Trento A et al. (2003) Major changes in the G protein of human respiratory syncytial virus isolates introduced by a duplication of 60 nucleotides. Journal of General Virology 84:3115-3120

Yoshihara K et al. (2016) Molecular evolution of respiratory syncytial virus subgroup A genotype NA1 and ON1 attachment glycoprotein (G) gene in central Vietnam. Infection, Genetics and Evolution 45:437-446

Zhang T et al. (2018) Tracing the emerging genotypes of human respiratory syncytial virus in Beijing by evolution analysis of the attachment glycoprotein (G) gene. Infection, Genetics and Evolution 65:18-27

Zhang Y, Yuan L, Zhang Y, Zhang X, Zheng M, Kyaw MH (2015) Burden of respiratory syncytial virus infections in China: Systematic review and meta-analysis. Journal of global health 5

Zheng Y et al. (2017) Prevailing genotype distribution and characteristics of human respiratory syncytial virus in northeastern China. Journal of medical virology 89:222-233 


\section{Tables}

Table 1. Demographic characterization of patients included in this study 


\begin{tabular}{|c|c|c|c|c|c|c|}
\hline & \multicolumn{3}{|l|}{ All cases } & \multicolumn{3}{|c|}{ RSV+ cases } \\
\hline & Inpatient & Outpatient & Total & Inpatient & Outpatient & Total \\
\hline \multicolumn{7}{|l|}{ Sex } \\
\hline Male & 100 & 36 & 136 & 41 & 15 & 56 \\
\hline Female & 48 & 22 & 70 & 13 & 5 & 18 \\
\hline \multicolumn{7}{|l|}{ Unknown } \\
\hline Total & 148 & 58 & 206 & 54 & 20 & 74 \\
\hline \multicolumn{7}{|l|}{ Months } \\
\hline January 2018 & 28 & 1 & 29 & 3 & 2 & 5 \\
\hline February 2018 & 43 & 9 & 52 & 26 & 1 & 27 \\
\hline March2018 & 2 & 8 & 10 & 2 & 5 & 7 \\
\hline December2018 & 4 & 7 & 11 & 0 & 0 & 0 \\
\hline January2019 & 3 & 8 & 11 & 0 & 1 & 1 \\
\hline February2019 & 15 & 8 & 23 & 6 & 6 & 12 \\
\hline March2019 & 18 & 2 & 20 & 11 & 3 & 14 \\
\hline April2019 & 14 & 3 & 17 & 2 & 1 & 3 \\
\hline May2019 & 21 & 12 & 33 & 4 & 1 & 5 \\
\hline Total & 148 & 58 & 206 & 54 & 20 & 74 \\
\hline \multicolumn{7}{|l|}{ Age groups } \\
\hline $1-5$ months & 96 & 14 & 110 & 33 & 15 & 48 \\
\hline 6-11 months & 40 & 22 & 62 & 15 & 3 & 18 \\
\hline $12-24$ months & 12 & 22 & 34 & 6 & 2 & 8 \\
\hline Total & 148 & 58 & 206 & 54 & 20 & 74 \\
\hline \multicolumn{7}{|l|}{ Clinical data } \\
\hline Fever & 130 & 45 & 175 & 48 & 10 & 58 \\
\hline Sore throat & 6 & 1 & 7 & 1 & 0 & 1 \\
\hline Cough & 86 & 38 & 124 & 26 & 10 & 36 \\
\hline Dyspnea & 65 & 10 & 75 & 21 & 3 & 24 \\
\hline Runny nose & 103 & 48 & 151 & 26 & 18 & 44 \\
\hline
\end{tabular}




\begin{tabular}{|lllllll|} 
Nasal congestion & 122 & 53 & 175 & 42 & 18 & 60 \\
\hline Restlessness & 109 & 36 & 145 & 38 & 10 & 48 \\
\hline Sneezing & 71 & 48 & 119 & 22 & 14 & 36 \\
Nausea & 49 & 19 & 68 & 14 & 7 & 21 \\
Anorexia & 22 & 15 & 37 & 5 & 4 & 9 \\
\hline Pneumonia & 29 & 6 & 35 & 12 & 5 & 17 \\
\hline Bronchiolitis & 106 & 41 & 147 & 37 & 12 & 49 \\
\hline Respiratory distress & 32 & 4 & 36 & 15 & 2 & 17 \\
\hline cyanosis & 4 & 0 & 4 & 0 & 0 & 0 \\
\hline Seizure & 2 & 48 & 50 & 0 & 0 & 0 \\
\hline Diarrhea & 3 & 1 & 4 & 1 & 0 & 1 \\
\hline Coma & 2 & 0 & 2 & 0 & 0 & 0 \\
\hline Nursing strike & 2 & 3 & 5 & 0 & 2 & 2 \\
\hline
\end{tabular}

Table 2. Primer sequences target N and $\mathbf{G}$ genes of HRSV

\begin{tabular}{|llll|}
\hline \multicolumn{2}{|l|}{ RSV G gene amplification, primers and products } & & \\
\hline primers & Sequence & Primer usage & Tm \\
\hline GPA & 5' GAAGTGTTCAACTTTGTACC3' $^{\prime}$ & Initial & 52.36 \\
\hline nRSAG & 5'TATGCAGCAACAATCCAACC3' $^{\prime}$ & Nested & 56.68 \\
\hline F1 & 5'CAACTCCATTGTTATTTGCC3' & Initial and nested & 53.09 \\
\hline RSV N gene amplification, primers and products & & \\
\hline primers & Sequence & Primer usage & Tm \\
\hline VrsP1 & 5' GGA ACA AGT TGT TGA GGTTTA TGA ATTGC 3' & Initial and nested & 61.83 \\
\hline VrsP2 & 5' TTC TGC TGT CAA GTC TAG TAC ACT GTA GT 3' & Initial & 63.25 \\
\hline Vrsi & 5' GGT GTA CCT CTG TAC TCT C 3' & Nested & 53.32 \\
\hline
\end{tabular}

\section{Figures}




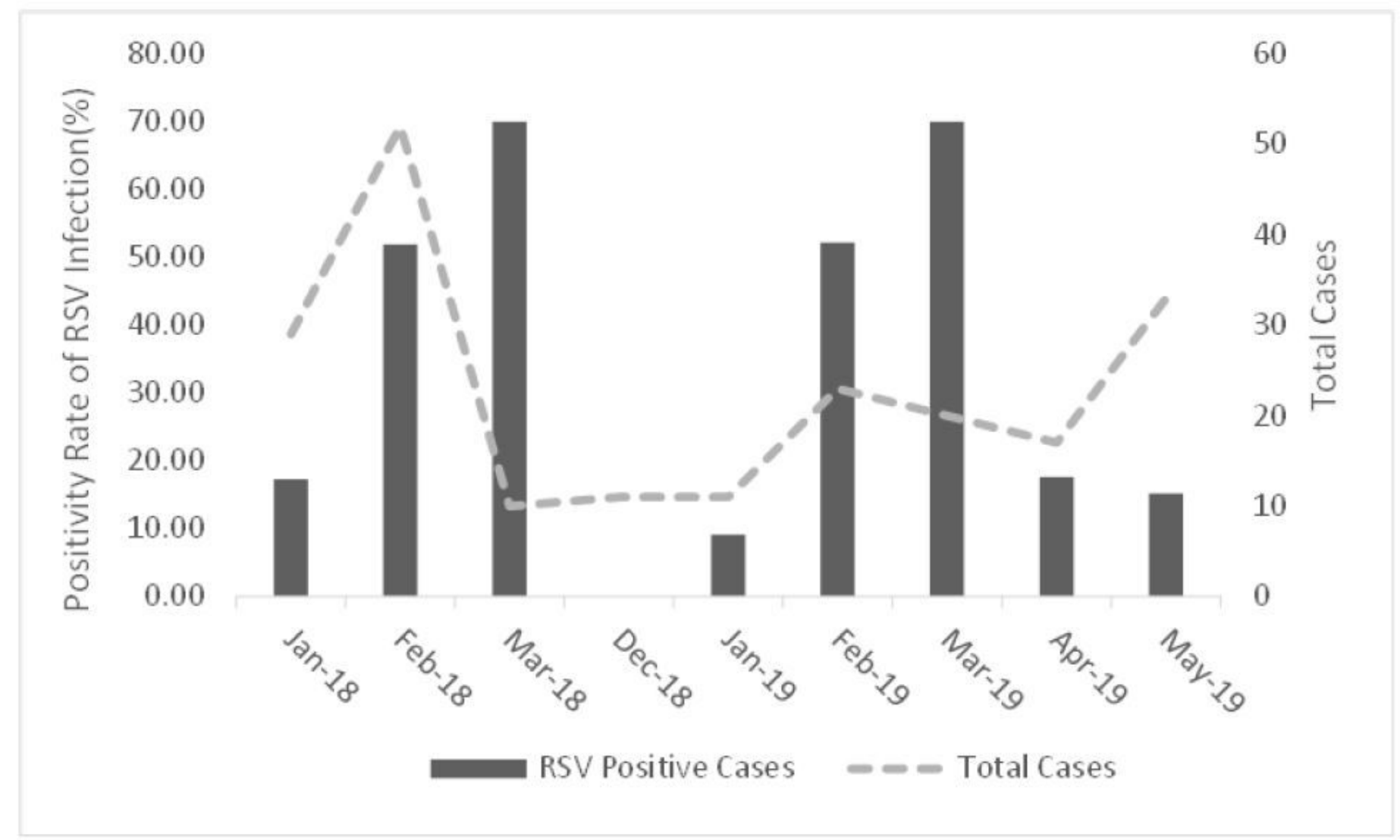

Figure 1

Monthly distribution of HRSV strains detected in 2018-2019 in Tehran, Iran. 

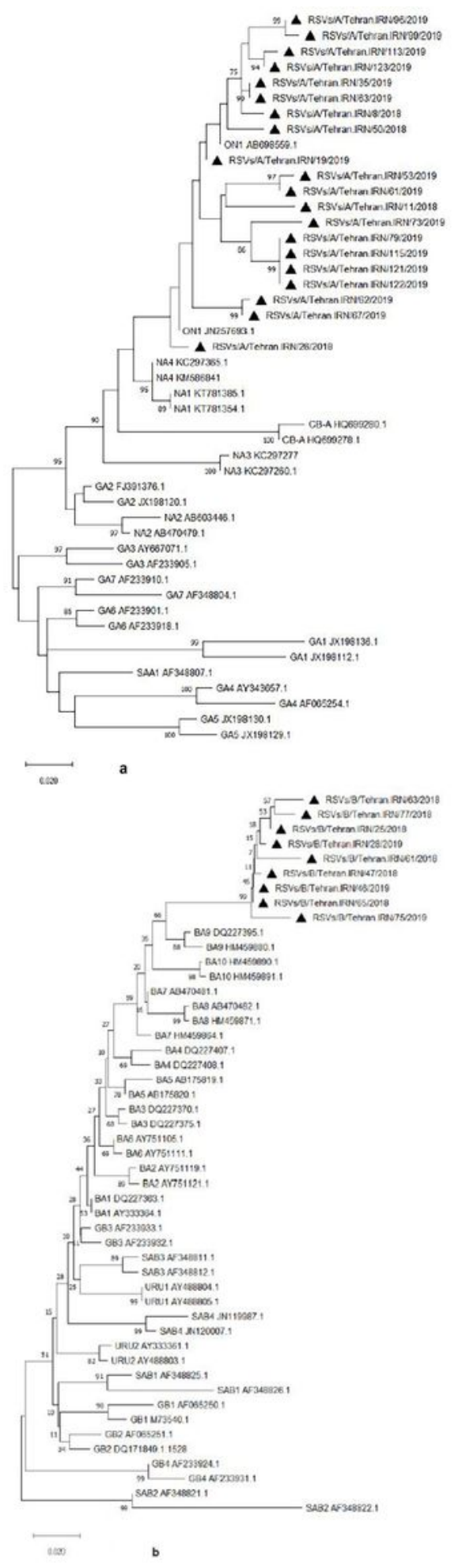

\section{Figure 2}

Phylogenetic tree of RSV A/RSV B strains and reference sequences of known genotypes. Phylogenetic trees for RSV A (A) and RSV B (B) strains were constructed with maximum-likelihood and Neighbor Joining method, respectively and Tamura-Nei model using MEGA $x$ software. RSV strains from Tehran/Iran are indicated by "solid triangle". Bootstrap values $>70 \% 70 \%$ are displayed at the branch nodes. 


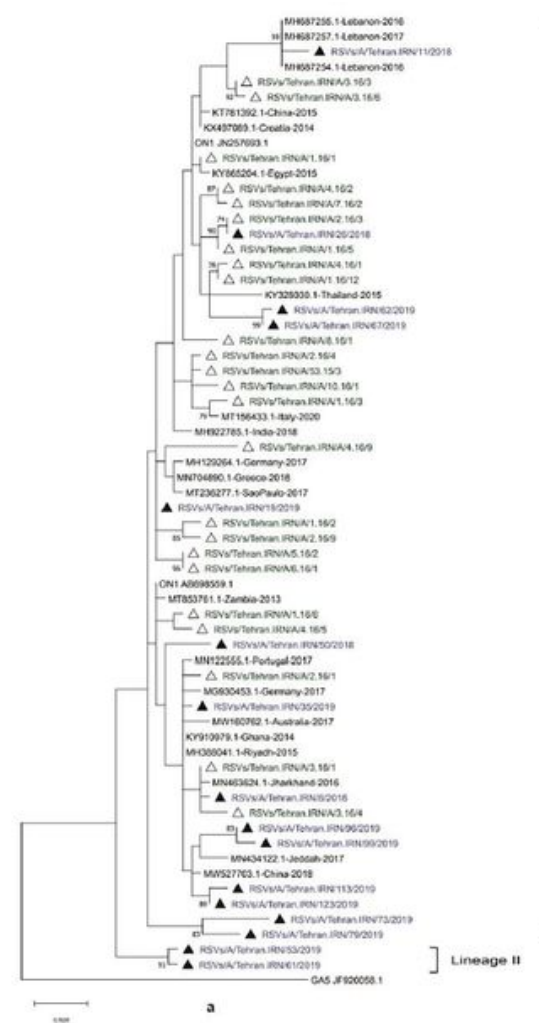

Lineage I

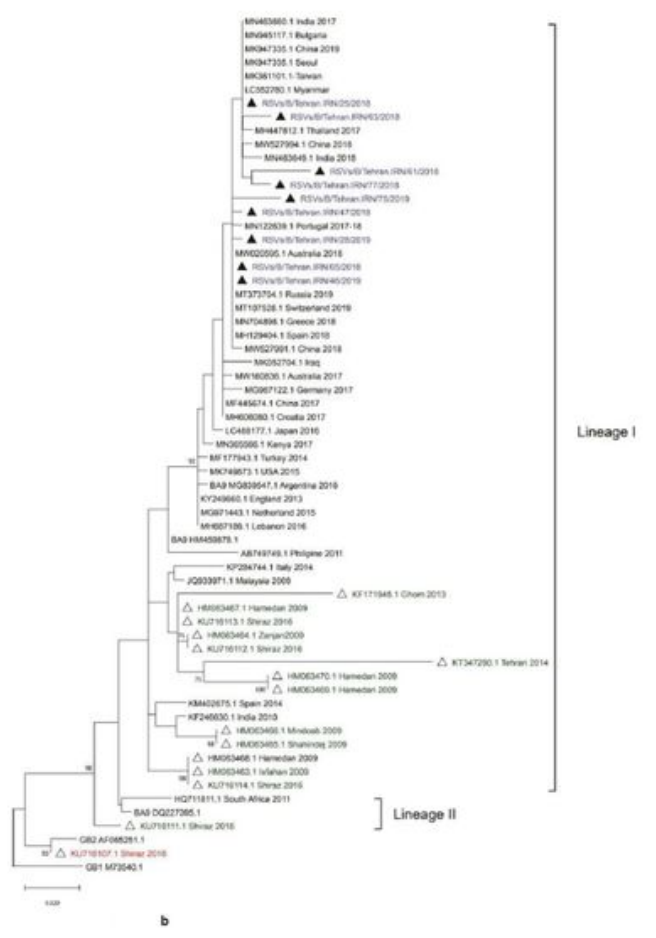

\section{Figure 3}

Phylogenetic tree of HVR2 G gene sequences of RSV-ON1 (A) and BA9 strains (B) from Iran (solid triangles and circles) compared with the sequences with high similarity (97\%-100\%) were constructed with maximum-likelihood and Tamura-Nei model for ON1 and HKY for BA9 strains using MEGA X software . bootstrap values $>70 \%$ are displayed at the branch nodes. The lineages $(\mathrm{I}, \mathrm{II})$ are indicated by Roman numerals. Our RSV strains from Tehran/Iran are indicated by "solid triangle" and isolates in the 
previous study in Tehran are indicated by "solid circles" for (A) and by solid circles and unfilled circles, respectively, for $(B)$.

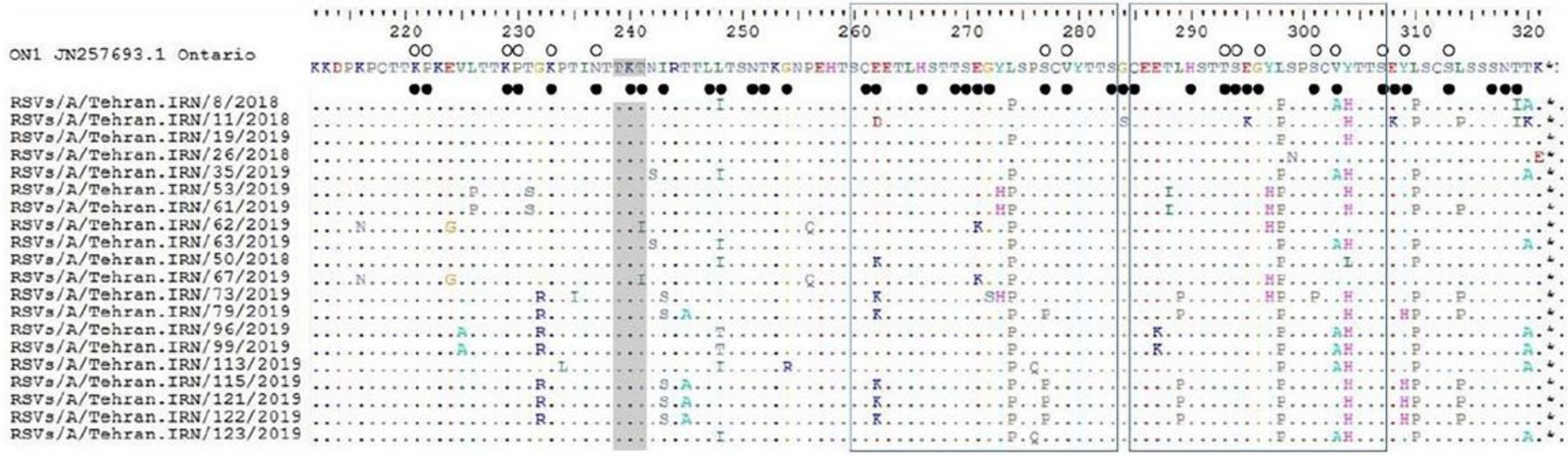

a

BA 2 АY33364.1

BA9 D2227395.1 RSVs/B/Tehran. IRN/25/2018 RSVs/B/Tehran. IRN/47/2018 RSVa/B/Tohran. IRN/63/201 RsVs/B/Tehran.IRN/61/201 RSVs/B/Tehran. IRN/6S/201 RSVs/B/Tehran. IRN/28/201 RsVs/B/Tehran.IRN/46/201 RSVs/B/Tehran. IRN/77/201 RSVo/B/Tohran. IRN/75/2019

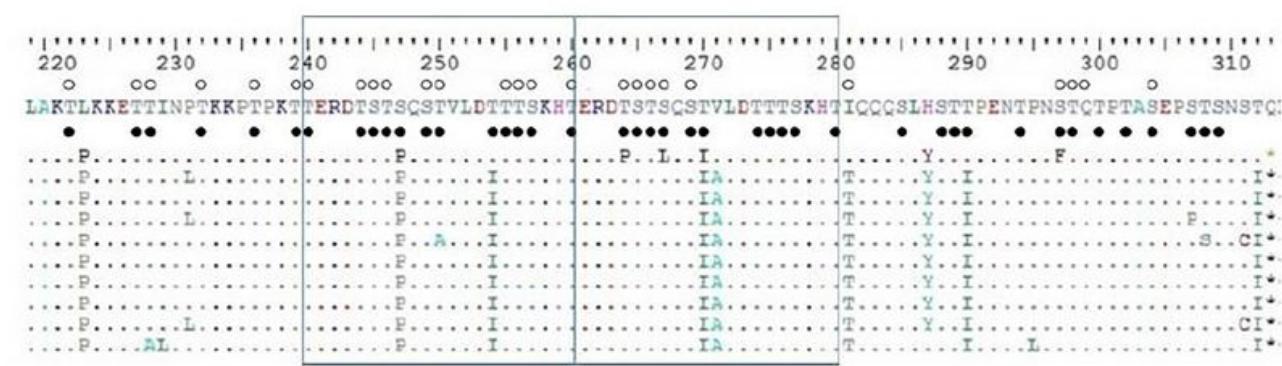

b

\section{Figure 4}

Amino acid alignments of the second high variable region of the $G$ genes. (A) RSV-A ON1 sequences alignment corresponding to positions 212 to 321 of the second hypervariable region of prototype ON1 genotype ON67-1210A (JN257693) (B) RSV-B BA9 sequences alignment corresponding to positions 219 to 313 of the $G$ protein of prototype BA genotype BA4128/99B (AY333364) and 217 to 310 of the $G$ protein of BA9 genotype BA/100/04 G (DQ227395.1). Predicted O-glycosylation sites of the reference ON1 and BA9 strain was shown by black dots and potential O-glycosylation sites of Iran strains are indicated by unfilled circles. Gray shading shows predicted N-glycosylation sites. Duplicated sites are shown by the outlined rectangles.

\section{Supplementary Files}


This is a list of supplementary files associated with this preprint. Click to download.

- Declarationsection.rsv.archive.docx 